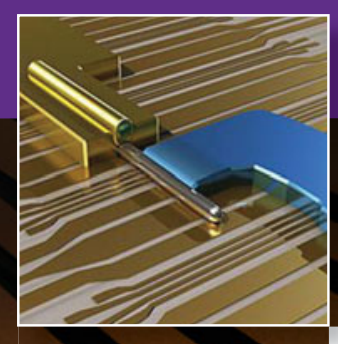

\title{
Materials issues for quantum computation
}

\author{
James N. Eckstein and Jeremy Levy, Guest Editors
}

The new field of quantum computing uses qubits (quantum bits) in place of classical bits to carry out certain types of computation. Physical systems that act as qubits encompass a wide range of technologies, from ions, to local defect states in crystals, and on to microelectronic devices addressable with wire interconnects. Materials issues arise in all of these, and this issue of MRS Bulletin describes how materials challenges and opportunities arise and have been used to make qubit-based quantum circuits using very different materials systems. In this overview article, we first review the universal ideas of how information is introduced and processed in a quantum computer. Comparing quantum to classical computers, for a given number of bits, the information content in a quantum computer is exponentially larger. But quantum computers face a daunting challenge: How do we keep the information from degrading and eventually disappearing? Maintaining the coherence of a quantum computer comes down to specific materials issues for all the approaches studied so far. Advances in materials design and processing have enabled enormous increases in performance, and we review the work described in each of the articles in this issue.

\section{Introduction}

In 1982, Richard Feynman wondered about the remarkable ability of quantum systems to compute their own time evolution. ${ }^{1}$ Could one harness the ability of quantum materials to solve the Schrödinger equation and create a fundamentally new class of computers?

Pioneering quantum computer scientists Peter Shor, Lov Grover, and others brought into focus the question of what a quantum computer can achieve that is new and different from ordinary ("classical") computers. Shor's algorithm ${ }^{2}$ provides an exponential speedup in the factoring of large integers, a capability that can efficiently defeat the Rivest-Shamir-Adleman (RSA) public key encryption scheme ${ }^{3}$ that is widely used on the Internet. Grover demonstrated ${ }^{4}$ how a quantum database could enable quadratic speedup in search queries. Lloyd proved the correctness of Feynman's original conjecture ${ }^{5}$ regarding quantum simulation, setting the stage for efficient quantum simulation of materials.

In a quantum computer, information is stored in quantum bits or "qubits," which can be thought of as a pair of quantum states that are part of "artificial atoms" made by the quantum mechanic. There are various ways of making these artificial atoms, and several of them are described in this issue of $M R S$ Bulletin. They range from ions, which are atomic systems, to devices made from billions of atoms such as quantum dots and Josephson junctions, which under the right circumstances behave like atomic systems. The internal states of these objects are described quantum mechanically, and for an $\mathrm{N}$-qubit system, the information is contained in the $\mathrm{N}$-body wave function. In most designs, the qubit is formed from only two quantum states out of the many states of the artificial atom that exist, so a qubit is a quantum two-level system. More specifically, the information processed in a quantum computer is contained in the quantum-mechanical amplitudes of each of the $N$-body, two-level system basis states. These are $2^{N}$ complex numbers. Such a system of $N$ qubits forms the memory of the quantum computer, and this memory is transformed by physical interactions that must be precisely controlled. To process this information, qubits are made to interact with each other via a pattern of classical control signals (e.g., electromagnetic fields) that couple the qubits and execute quantum logic operations. Such a quantum algorithm results in the evolution of all quantum information. The fact that controlling the interactions between $N$ qubits drives the evolution of $2^{N}$ complex coefficients is central to why quantum computing is so powerful, at least at some tasks.

However, the information stored in these qubits is delicate. The complex amplitudes that specify a qubit can be corrupted 
in two principal ways. First, many qubits are formed from the ground state and an excited state of a physical system. In this case, the energy relaxation mechanism to the ground state that leads to the radiative lifetime of the system causes the state of the qubit to decay, resulting in the loss of quantum information. The second way in which quantum information can be lost is via dephasing. Interaction of a qubit with the environment leads to additional relaxation channels that affect the phase as well as the lifetime of the qubit state. Essentially, if the qubit interacts with residual environmental degrees of freedom, for example other unaccounted for two-level systems, this will cause the qubit's phase to change in a seemingly random manner even if the magnitudes of the amplitudes remain constant. Reducing ways in which the qubit interacts with its physical environment is an important objective of every quantum technology.

Over the last 15 years, the field of quantum computation has grown from a highly specialized and arcane branch of physics to one of the most challenging and demanding frontiers of materials science, engineering, and emerging technology. While there are still no "useful" scalable quantum computers, progress has been sustained, with several strong candidate architectures. Many of the challenges in developing a quantum computer relate to issues that are naturally suited for materials science.

In this issue of MRS Bulletin, leading researchers in various sub-disciplines of quantum information science describe specific materials-related challenges in constructing robust, long-lived quantum bits - qubits - and requisite quantum logic. These challenges can differ radically from classical information technologies due to the unique demands placed by quantum computation. This new field of quantum computation creates unique research opportunities for researchers with a deep expertise and understanding of these materials. It is hoped that this Introductory article will help to channel the expertise of the materials community to address challenges faced by quantum computing researchers.

Before we describe in detail the various approaches and relevant materials for quantum computation, it is worth giving a quick functional overview of how a quantum computer operates. Excellent reviews of quantum information and computation are readily available. ${ }^{6-8}$ Here we will focus on the essential aspects that one needs to know to understand the relationship to materials development.

It is helpful to compare the operation of a quantum computer with a classical one, for in the end, both are expected to provide "answers" to computational problems. A "traditional" quantum computer should be "invisible" to the user in that it provides the answer to a problem that an ordinary computer is able to compute, but presumably with fewer computational steps. Such is certainly the case for Shor's and Grover's algorithms, but for most applications (e.g., sorting tasks), there is no known speedup.

\section{Quantum bits are materials}

The essential link between information and materials was continually stressed by Rolf Landauer: "Information is physical."
DiVincenzo and Loss argued that "quantum information is physical," 10 meaning that the quantum generalization of information only further highlights the inextricability of the information from its physical embodiment. In the same article, they laid out five important criteria for quantum computation. (A later article published ${ }^{11}$ in 2000 , devoted exclusively to quantum computing criteria, ${ }^{10}$ adds two requirements for quantum information transmission.) These requirements, highlighted in the sidebar, quickly became "marching orders" for a wide range of experimental proposals, many of which are described in this issue.

This basic set of criteria rest on the ability to rapidly and accurately manipulate $2^{N}$ complex coefficients, presenting unique challenges for the materials used to host quantum information, particularly those associated with dephasing and errors that creep in that are intrinsic to quantum computing.

The issue is not whether phase information is lost but rather how fast. This is generally referred to as "dephasing," a term familiar from nuclear magnetic resonance (NMR) studies of inhomogeneous systems. In fact, some of the earliest demonstrations of quantum computing algorithms were performed using NMR. ${ }^{12,13}$ The dephasing time is traditionally represented by $T_{2}$, while energy relaxation is governed by another time scale, $T_{1}$.

An important component of any realistic quantum computer is a method for "quantum error correction." Quantum errors arise from unwanted interference in the environment, leading to dephasing and other unwanted disturbances or "quantum entanglement"14 with the environment surrounding the qubits. Quantum error correction is challenging because measurements of a quantum state in general disrupt the delicate superpositions that they are supposed to protect. The first quantum error correction scheme was invented ${ }^{15}$ by Peter Shor (who also conceived the quantum factoring algorithm ${ }^{2}$ ). Amazingly, Shor employed "spooky" quantum entanglement inherent in quantum mechanics ${ }^{16}$ to correct quantum errors without directly measuring the quantum state. (A complete history of the early development of quantum computing can be found in Reference 17.) Depending on the quantum computing architecture, various error thresholds are required. Some of the most sophisticated codes (e.g., surface codes) ${ }^{18}$ require hundreds or thousands of physical qubits to stabilize a single logical qubit. A truly stable qubit, able to persist in a given quantum superposition over an indefinite period of time (with the aid of quantum error correction), has not yet been demonstrated.

\section{Materials metrics: The eye of the beholder}

For the material scientist, one aspect that is particularly refreshing about the field of quantum computation is the often contrasting relationship between traditional metrics for information materials (e.g., complementary metal oxide semiconductor [CMOS] or magnetic storage) and those for quantum computing applications. In most applications, for instance, defects are undesirable, leading to reduced mobility and other degraded 


\section{Requirements for quantum computing}

a

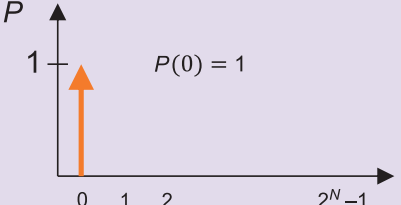

b

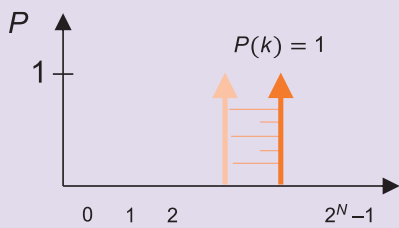

C

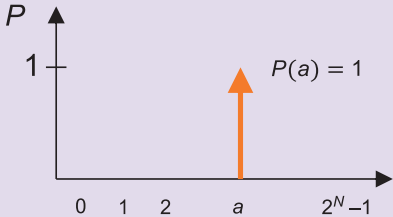

d $P$

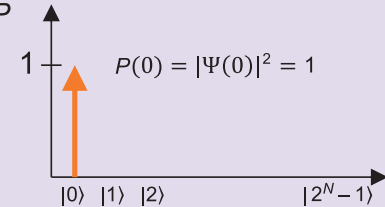

e $P$

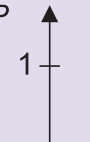

$i \hbar \frac{d|\Psi\rangle}{d t}=\widehat{H}(t)|\Psi\rangle$

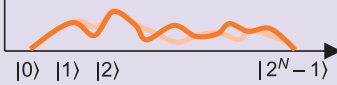

f $P$

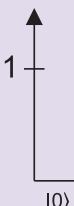

The first four requirements are "quantum" generalizations of requirements for ordinary computation - simply adding the word "quantum" to known requirements for universal (classical) computation. The fifth requirement, quantum coherence, represents a radical departure from the classical bit. The distinction between $|0\rangle$ and $|1\rangle$ is common to both classical and quantum computation, but the notion of quantum superposition, that $|0\rangle+|1\rangle$ and $|0\rangle-|1\rangle$ represent distinct quantum states that are non-orthogonal to $|0\rangle$ and $|1\rangle$, means that there is more "internal" information stored in one (or many) quantum bits. A single quantum bit can be specified by two complex coefficients $a_{0}$ and $a_{1}:\left|\Psi_{1}\right\rangle=a_{0}|0\rangle+a_{1}|1\rangle$, where $|0\rangle$ and $|1\rangle$ are distinct quantum states of the physical qubit. The most general $N$-qubit quantum state requires $2^{N}$ such complex numbers $\left\{a_{0}, a_{1}, \ldots, a_{2^{N}-1}\right\}$ to be specified:

A scalable quantum computer must possess:

1. Scalable quantum memory.

2. Ability to initialize qubits.

3. Universal quantum gates.

4. Qubit readout.

5. Long coherence times for the qubits.

6. Interconversion between stationary and "flying" (e.g., photonic) qubits.

7. Faithful transmission of flying qubits.
Much of the power of quantum computers is derived from this "internal" information. To represent even a modest number of qubits (300 or so) classically, using one atom per bit, would require more atoms than exist in the known universe. properties. However, defects in solids form the starting point for some solid-state quantum bits. The nitrogen-vacancy (NV) center in diamond (see the February 2013 issue of MRS Bulletin) (and related defects in silicon carbide) has exceedingly long coherence times that make it useful for long-lived room-temperature quantum memory. Quantum tunneling through oxide barriers can be problematic for CMOS logic; but controlled quantum tunneling is an important and often essential part of quantum logic. On the flip side, two-level "fluctuators" residing in dielectrics used in Josephson junctions, unnoticeable for conventional applications, have a profound effect on coherence times in superconducting qubits. As the saying goes, "beauty is in the eye of the beholder."

Depending on the architecture and proposal, sometimes enormous precision over the placement of single defects is required. In general, proposals that utilize solid-state materials to create and manipulate qubits often require excruciating control over material properties. Literally, single dopant atoms can constitute a qubit. Not all solid-state proposals require such control though. For example, superconducting qubits are typically quite "big" (i.e., millimeter-scale), involving superconducting transmission lines that are required to reduce 
the sensitivity of "transmon" qubits to charge noise. Extended even further into space are ion trap quantum computing architectures. In this case, the qubits are composed of internal degrees of freedom of single ions. But it is the manner in which these ions are trapped and made to interact that introduces many materials challenges.

\section{Materials paradigms for quantum computing}

This issue of MRS Bulletin highlights the materials issues associated with five different approaches to quantum computation. These span an interesting axis of coherence and coupling. Generally, systems that are hard to couple to do not experience as much dephasing from inhomogeneous environmental noise sources, and this causes their coherence times to be longer. On the other hand, systems that are easy to couple experience just the opposite. Qubits based on ions in traps are an example of the former. They are isolated and have relatively long coherence times, but they are also more difficult to couple together. On the other end of the axis, superconducting qubits based on Josephson junctions can be coupled together in circuits made using standard lithographic and microelectronic techniques. However, they suffer from noise picked up on the transmission lines, and this leads to relatively much shorter coherence times. In all cases, the precision of the materials used to make the qubits is paramount to solving these two contradictory requirements. The key materials basis for each of the five computing paradigms, listed in Figure 1, are reviewed in order to provide a primer for non-experts in each arena.

\section{Qubits from quantum dots}

One of the most powerful approaches to solid-state quantum computation involves the use of two-dimensional electron gases that are gated so as to confine single electrons and their spins. The technology that allows single electrons or holes to be confined in nanometer-scale regions of semiconductors has advanced only in the last decade or so, leading to startling scientific and technological demonstrations.

The twofold spin degeneracy of a single electron, which can be controlled now with exquisite precision, offers a natural basis for a quantum bit. The so-called "Loss-DiVincenzo" approach to spin-based quantum computing, named after two authors of a seminal theory proposal, ${ }^{10}$ has guided this approach to experimental quantum computing for the last 15 years.

Eriksson et al., in this issue, survey efforts in both Group III-V and Group IV semiconductor structures, outlining several opportunities for materials research. Of the two, the III-V systems are more thoroughly investigated ${ }^{25}$ but have features such as strong hyperfine coupling to nuclear spins that are not present in isotopically purified SiGe systems. The SiGe system is not as extensively developed as the GaAs/AlGaAs system but is seeing rapid progress. Recently, "single-shot" readout of individual spins in $\mathrm{SiGe}$ quantum dots has been achieved, ${ }^{26}$ which is an essential component in the functioning of any quantum computer. There are many materials issues in the SiGe system, such as lattice mismatch, ${ }^{27}$ valley degeneracies, ${ }^{28}$ and relaxation via misfit dislocations ${ }^{29}$ Both $\mathrm{SiGe}$ and AlGaAs exhibit high sensitivity to charge fluctuation noise, some of which can be minimized by proper device layer engineering.

\section{Qubits from defects}

The search for defect states in solids with the capacity to store and manipulate quantum information represents a vibrant area of research. In particular, recent advances bring to focus the predictive power of modern computational techniques, most notably density functional techniques, in searching for new material hosts for long-coherence qubits. In their article, Gordon and co-authors review the NV center in diamond, ${ }^{30,31}$ which holds promise for a host of applications, including quantum computation. The attractiveness of this system originates from the long-lived quantum coherence, which can be initialized, acted upon, and measured using readily available techniques. ${ }^{32}$ A particularly exciting feature of this class of defects is the persistence of long coherence times even at room temperature. ${ }^{33}$ Single NV centers can be patterned on demand, and much like atomic defects, they have highly reproducible properties.

Motivated by success with diamond, Gordon et al. describe the theory-driven search for defects in other material hosts. Taking advantage of the latest computational techniques, ${ }^{34-37}$ the authors describe several interesting alternate materials. One of them, a di-vacancy center in $\mathrm{SiC}$, has been found and shares many of the attractive features of the diamond NV center. ${ }^{38,39}$ 
There are likely to be other such defects that are waiting to be discovered that have attractive features for quantum computation. The approach taken here highlights the important role played by computational methods in advancing the materials science relevant to quantum computation.

\section{Topological qubits made with semiconductor nanowires}

In their article on qubits formed in semiconductor nanowires, Frolov et al. describe both the growth of precisely formed nanowires as well as how qubits can be made by using them. Gold nanoparticles dispersed on a substrate surface provide nucleation sites and catalyze the formation of nanowires in chemical vapor deposition. ${ }^{40}$ With two dimensions of confinement already formed by the wire geometry, quantum dot arrays can be made with linear arrangements of electrostatic gates that can be used to both define the size of the dot as well as the coupling between dots. When combined with the superconducting proximity effect and an appropriately chosen magnetic field, quantum wires made of semiconductors with large spin-orbit coupling have been proposed to give rise to the exotic Majorana fermion.$^{41}$ Indeed, in a pioneering experiment, the authors of this article showed spectroscopic evidence for the formation of Majorana fermions under exactly the right conditions for them to be formed. ${ }^{42}$ The benefit of their application to quantum computing comes from the nature of the Majorana fermion itself. Two of these objects, which are necessarily physically separated in different locations in space, arise from one electron state, and a topological qubit resistant to dephasing by local perturbations can be made using them. ${ }^{43}$ In a topological qubit, the location of the Majorana fermion is the quantum variable, as illustrated in Figure 2. Since many of the problems leading to decoherence and decay arise from the interaction of a qubit with local fields, by using a delocalized quantum mechanical state, some level of topological protection against loss of quantum information is obtained.

\section{Superconducting qubits}

Superconducting qubits are lithographically fabricated electrical circuits comprising inductors, capacitors, Josephson junctions, and interconnects. In their article, Oliver and Welander review superconducting qubits, their decoherence due to materials defects, and the tremendous improvements that have been achieved over the past decade.

At the core of all superconducting qubits is the Josephson tunnel junction, which is a weak link between two superconductors mediated by electron tunneling. ${ }^{44}$ When cooled to temperatures accessible to a ${ }^{3} \mathrm{He} /{ }^{4} \mathrm{He}$ dilution refrigerator ( 20 millikelvins), these junctions exhibit quantized energy levels and behave as "artificial atoms." For example, the quantum behavior of a Josephson phase qubit is due to quantum fluctuations in the phase difference between the two superconducting electrodes, and this is visible if the experimental environment is sufficiently quiet..$^{45}$ The wave function of the ground state at zero current is a symmetric Gaussian function of the phase difference, and the wave function of the first excited state is an antisymmetric function of the phase difference. This is illustrated in Figure 2. Other qubit designs are also possible, for example flux qubits that consist of a superconducting loop in which one or more Josephson junctions are placed. Flux states play the role of phase in these qubits.

Although superconducting qubits are macroscopic in size, containing about a billion atoms, many of the dynamical degrees of freedom are "frozen out" by the superconducting gap and the associated low temperature operation. Nonetheless, materials (or materials-related) defects remain. These are spurious two-level systems that interact with the qubit and localized surface electron states that can hold one electron spin. Such defects may cause energy decay and/or dephasing of the qubit, limiting their effective coherence times and, ultimately, their utility in quantum information science and technology applications ${ }^{46}$ In their article, Oliver and Welander

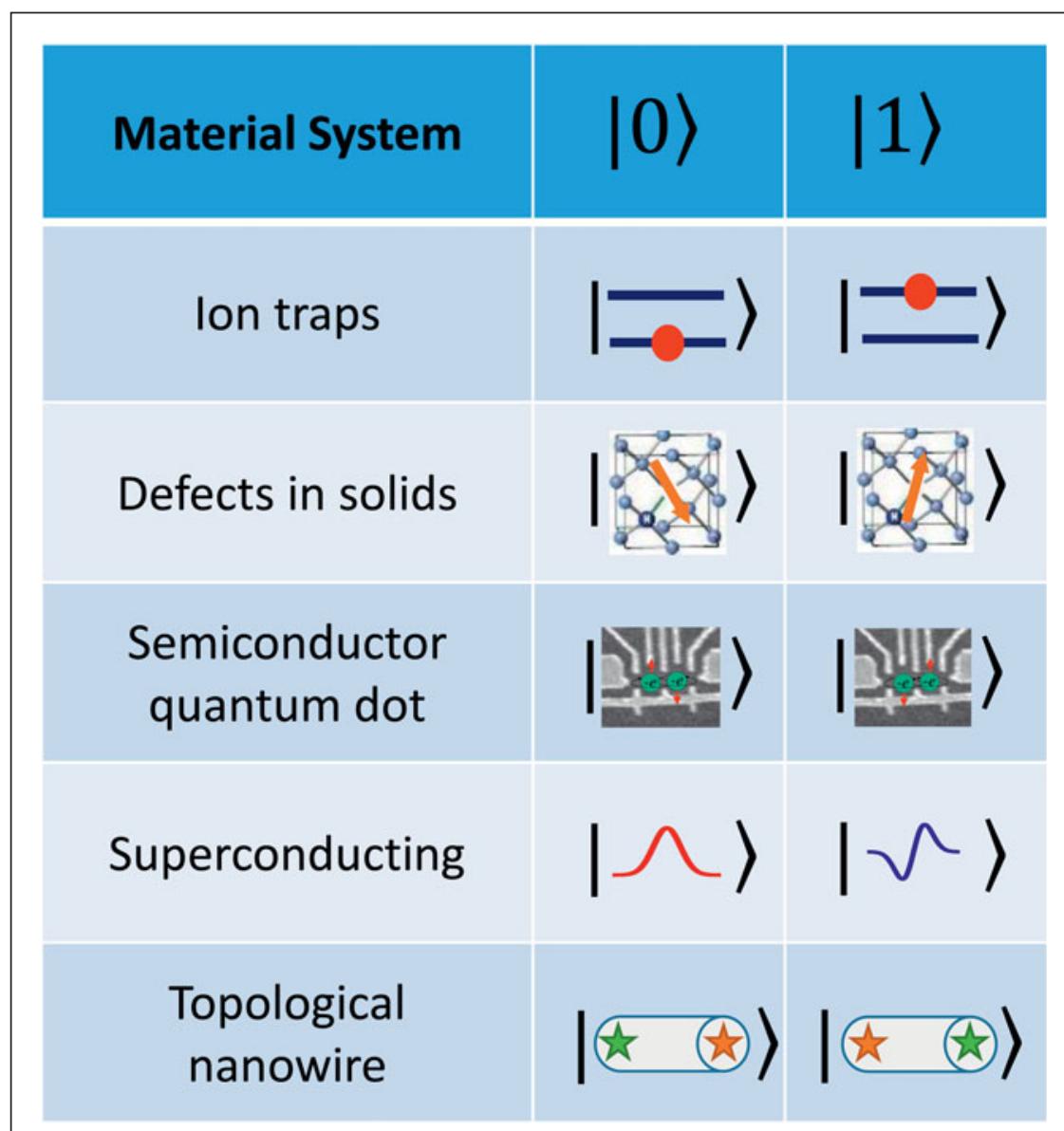

Figure 2. Qubit states for five material systems. 
review superconducting qubits, associated decoherence due to materials defects, and the tremendous improvements that have been achieved over the past decade.

\section{Qubits based on ions in ion traps}

Electromagnetic confinement of individual ions ("ion traps") represent a highly promising approach to the development of quantum information technology. ${ }^{47}$ Here, the quantum state is a combination of the hyperfine electronic state of the ion and its center-of-mass motional state. ${ }^{48}$ The ions are held in a harmonic confining potential provided by voltages applied to electrodes near the ion. The electrode geometry is a linear quadrupole much like what is found in mass analyzers in UHV systems, and ac voltages provide a nonlinear confining (pondermotive $)^{49}$ force to keep the ions nearly on-axis. Quantum information is contained in the superposition of the two hyperfine states chosen to be used as 0 and 1 . This is shown schematically in Figure 2. When the ion is excited from the motional ground state, this electronic superposition is changed. This process is used to bring two ions into close enough proximity that they interact. However, this also happens when local defect states on the surface of the electrodes that provide the confining potential for the ions become charged. This causes spurious electric fields that act on the ion. ${ }^{50} \mathrm{~A}$ sudden electric field can excite the ion into higher energy translational states. This is referred to as anomalous heating with respect to the translational motion of the ions. It causes decoherence due to the unintentional mixing of the hyperfine states as a result of the motion in the confining potential.

Understanding how this happens has been an important question for groups using ion traps to make qubits. Hite et al. describe in this issue experiments to study how motional excitation is driven by surface defects and how this leads to quantum decoherence. The amount of anomalous heating observed is substantially greater than that attributable to Johnson noise, so other surface dynamics must be in play. The authors report that by combining sophisticated surface analysis with in situ surface processing, a substantial reduction in the heating rate has been obtained. They outline the value of such improvements to a variety of other fundamental experiments.

\section{Other quantum computer paradigms}

The overview presented in this issue, while extensive, is far from comprehensive. Many important materials systems are not covered, not because they are unimportant but perhaps because they do not fit neatly into the five focus areas chosen. One important and highly influential proposal is the Kane quantum computer. ${ }^{51}$ Here, the qubit is formed from electron and nuclear spins associated with single phosphorous donors in silicon. At the time it was proposed, the technology for isolating and producing such structures seemed far from reality. But in recent years the technical and materials challenges are being met, and this approach remains vital.

There are also other less mainstream approaches to quantum computation involving, for example, electrons trapped in surface states of helium, ${ }^{52}$ exciton-polariton states,${ }^{53}$ linear optical quantum computing, ${ }^{54}$ and others that are not covered in this issue. A related area of materials research focuses on the bottom-up synthesis of new quantum materials using ultracold neutral atoms trapped in standing waves of light. The so-called "cold atom Hubbard toolbox," ${ }^{55}$ for instance, refers to a largescale effort to develop a new type of quantum materials science where materials and physical couplings are engineered and precisely controlled, with the goal of simulating new phases of matter and ultimately serving as a tool for the guided discovery of new materials.

The private manufacturer, D-Wave, advertises that "Quantum computing has arrived," and markets a second-generation quantum computing system with a "512-qubit processor chip" consisting of pair-wise coupled superconducting flux qubits. ${ }^{56}$ The technology that D-Wave offers is fundamentally different from the architectures described previously, but is also likely to be susceptible to materials challenges similar to those for superconducting qubits. Recent work shows that the D-Wave quantum computers are likely what are known as "adiabatic quantum computers" that anneal the input state. Furthermore, there have been recent measurements claiming speed-up of various computational problems over classical computers. ${ }^{57}$ It is also worth noting that there are still disputes about all of these claims. Nonetheless, on the basis of work-to-date, D-Wave has sold their quantum computers to a number of applied research laboratories, and work is under way to evaluate their computing performance.

\section{Summary}

This issue presents review articles that describe five different approaches to quantum computing and the special materials issues affecting quantum coherence that arise in each of them. Preserving and maintaining the quantum information in qubits, entangling the component qubits by controllably causing the qubits to interact, and reading out the final wave-function probability distributions are challenges they all face. The materials that qubits and quantum circuits are made from have a strong influence on how these are accomplished. Defects that act as two-level systems play a central role. When they can be controlled, they can be used as qubits themselves. Otherwise, they are a source of noise and a cause of decoherence. The articles in this issue explain how this work has been driven by the performance of quantum devices and circuits and how advances in materials science have contributed to the development of the field of experimental quantum information.

\section{References}

1. R.P. Feynman, Int. J. Theor. Phys. 21, 467 (1982).

2. P.W. Shor, SIAM J. Comput. 26, 1484 (1997)

3. R.L. Rivest, A. Sharmir, L. Adleman, Commun. ACM 21, 120 (1978).

4. L. Grover, Proceedings of 28th Annual ACM Symposium on Theory of Computing (STOC), 212 (1996).

5. S. Lloyd, Science 273, 1073 (1996)

6. M.A. Nielsen, I.L. Chuang, Quantum Computation and Quantum Information (Cambridge University Press, Cambridge, UK, 2000).

7. B. Ruggiero, P. Delsing, C. Granata, Y. Pashkin, P. Silvestrini, Quantum Computation in Solid State Systems (Springer, New York, 2006). 
8. N.D. Mermin, Quantum Computer Science: An Introduction (Cambridge University Press, Cambridge, UK, 2007).

9. R. Landauer, Physics Today 44(5), 23 (1991).

10. D.P. DiVincenzo, D. Loss, Superlattices Microstruct. 223, 419 (1998).

11. D.P. DiVincenzo, Fortshritte der Physik 48, 771 (2000).

12. L.M.K. Vandersypen, M. Steffen, G. Breyta, C.S. Yannoni, M.H. Sherwood, I.L. Chuang, Nature 414, 883 (2001).

13. J.A. Jones, Prog. Nucl. Magn. Reson. Spectrosc. 59, 91 (2011).

14. E. Schrödinger, M. Born, Mathematical Proceedings of the Cambridge Philosophical Society 31, 555 (1935).

15. P.W. Shor, Phys. Rev. A 52, R2493 (1995).

16. A. Einstein, B. Podolsky, N. Rosen, Phys. Rev. 47, 777 (1935)

17. H.E. Brandt, Prog. Quantum Electron. 22, 257 (1998).

18. A.G. Fowler, M. Mariantoni, J.M. Martinis, A.N. Cleland, Phys. Rev. A 86, 032324 (2012).

19. Wikipedia, "Blochsphere," available at http://en.wikipedia.org/wiki/File: Blochsphere.svg.

20. L. DiCarlo, J.M. Chow, J.M. Gambetta, L.S. Bishop, B.R. Johnson, D.I. Schuster, J. Majer, A. Blais, L. Frunzio, S.M. Girvin, R.J. Schoelkopf, Nature, 460, 240 (2009).

21. M. Eriksson, Silicon Nanostructures, available at http://uw.physics.wisc.edu/ eriksson/images/research/nanostructures.jpg.

22. B. Yirka, Phys. Org. (2013), available at http://phys.org/news/2012-04-evidencemajorana-fermions.html.

23. Luleå University of Technology, "Quantum Bits in Quantum Computers," available at http://www.Itu.se/research/subjects/Tillampad-fysik/Forskningsprojekt/ Elektronstrukturteori/Kvantbitar-i-kvantdatorer-1.105526?l=en.

24. Softpedia, "New Ion Trap May Lead to Large Quantum Computers," available at http://news.softpedia.com/news/New-Ion-Trap-May-Lead-To-LargeQuantum-Computers-29646.shtml.

25. H.L. Störmer, R. Dingle, A.C. Gossard, W. Wiegmann, M.D. Sturge, Solid State Comm. 29, 705 (1979).

26. C.B. Simmons, J.R. Prance, B.J. Van Bael, T.S. Koh, Z. Shi, D.E. Savage, M.G. Lagally, R. Joynt, M. Friesen, S.N. Coppersmith, M.A. Eriksson, Phys. Rev. Lett. 106, 156804 (2011).

27. P. Mooney, Mat. Sci. \& Eng. R-Reports 17, 105 (1996).

28. K. Lai, W. Pan, D.C. Tsui, S. Lyon, M. Mühlberger, F. Schäffler, Phys. Rev. Lett. 96, 076805 (2006).

29. E. Kasper, H.J. Herzog, Thin Solid Films 44, 357 (1977)

30. G. Davies, M.F. Hamer, Proc. R. Soc. Lond Ser-A 348, 285 (1976).

31. A. Gali, E. Janzén, P. Deak, G. Kresse, E. Kaxiras, Phys. Rev. Lett. 103 186404 (2009).
32. N.B. Manson, J.P. Harrison, M.J. Sellars, Phys. Rev. B. 74, 104303 (2006). 33. D. Toyli, D.J. Christie, A. Alkauskas, B.B. Buckley, C.G. Van de Walle, D.D. Awschalom, Phys. Rev. X 2, 031001 (2012).

34. C.G. Van de Walle, J. Neugebauer, J. Appl. Phys. 95, 3851 (2004)

35. J. Heyd, G.E. Scuseria, M. Ernzerhof, J. Chem. Phys. 118, 8207 (2003).

36. J. Heyd, G.E. Scuseria, M. Ernzerhof, J. Chem. Phys. 124, 219906 (2006)

37. C.G. Van de Walle, A. Janotti, Phys. Status Solidi B 248, 19 (2011).

38. A.L. Falk, B.B. Buckley, G. Calusine, W.F. Koehl, V.V. Dobrovitski, A. Politi, C.A. Zorman, P.X.-L. Feng, D.D. Awschalom, Nat. Commun. 4, 1819 (2013)

39. W.F. Koehl, B.B. Buckley, F.J. Heremans, G. Calusine, D.D. Awschalom, Nature 479, 84 (2011).

40. H.J. Joyce, J. Wong-Leung, Q. Gao, H.H. Tan, C. Jagadish, Nano Lett. 10 $908(2010)$

41. R.M. Lutchyn, J.D. Sau, S. Das Sarma, Phys. Rev. Lett. 105, 077001 (2010) 42. V. Mourik, K. Zuo, S.M. Frolov, S.R. Plissard, E.P.A.M. Bakkers, L.P. Kouwenhoven, Science 336, 1003 (2012).

43. A. Stern, N.H. Lindner, Science 339, 1179 (2013).

44. T. Van Duzer, C. Turner, Principles of Superconducting Devices and Circuits (Prentice Hall, New Jersey, 1999).

45. J.M. Martinis, Quant. Info. Proc. 8, 81 (2009).

46. J. Clarke, F.K. Wilhelm, Nature 453, 1031 (2008)

47. R. Blatt, D.J. Wineland, Nature 453, 1008 (2008)

48. B. Blinov, D. Leibfried, C. Monroe, D.J. Wineland, Quant. Info. Proc. 3, 45 (2004).

49. Wikipedia, "Ponderomotive Force," available at http://en.wikipedia.org/ wiki/Ponderomotive force.

50. D.J. Wineland, C. Monroe, W.M. Itano, D. Leibfried, B.E. King, D.M. Meekhof, J. Res. Natl. Inst. Stand. Tech. 103, 259 (1998).

51. B.E. Kane, Nature 393, 133 (1998)

52. P.M. Platzman, M.I. Dykman, Science 284, 1967 (1999)

53. S. Puri, N.Y. Kim, Y. Yamamoto, "Exciton-Polariton Mediated Universal Quantum Computing," QM3C.5 (Optical Society of America, 2013).

54. P. Kok, W.J. Munro, K. Nemoto, T.C. Ralph, J.P. Dowling, G.J. Milburn, Rev. Mod. Phys. 79, 135 (2007)

55. D. Jaksch, P. Zoller, Ann. Phys. 315, 52 (2005)

56. R. Harris, M.W. Johnson, T. Lanting, A.J. Berkley, J. Johansson, P. Bunyk, E. Tolkacheva, E. Ladizinsky, N. Ladizinsky, T. Oh, F. Cioata, I. Perminov, P. Spear, C. Enderud, C. Rich, S. Uchaikin, M.C. Thom, E.M. Chapple, J. Wang, B. Wilson, M.H.S. Amin, N. Dickson, K. Karimi, B. Macready, C.J.S. Truncik, G. Rose, Phys. Rev. B 82, 024511 (2010).

57. S. Boixo, T. Albash, F.M. Spedalieri, N. Chancellor, D.A. Lidar, Nat. Commun. 4, 2067 (2012)

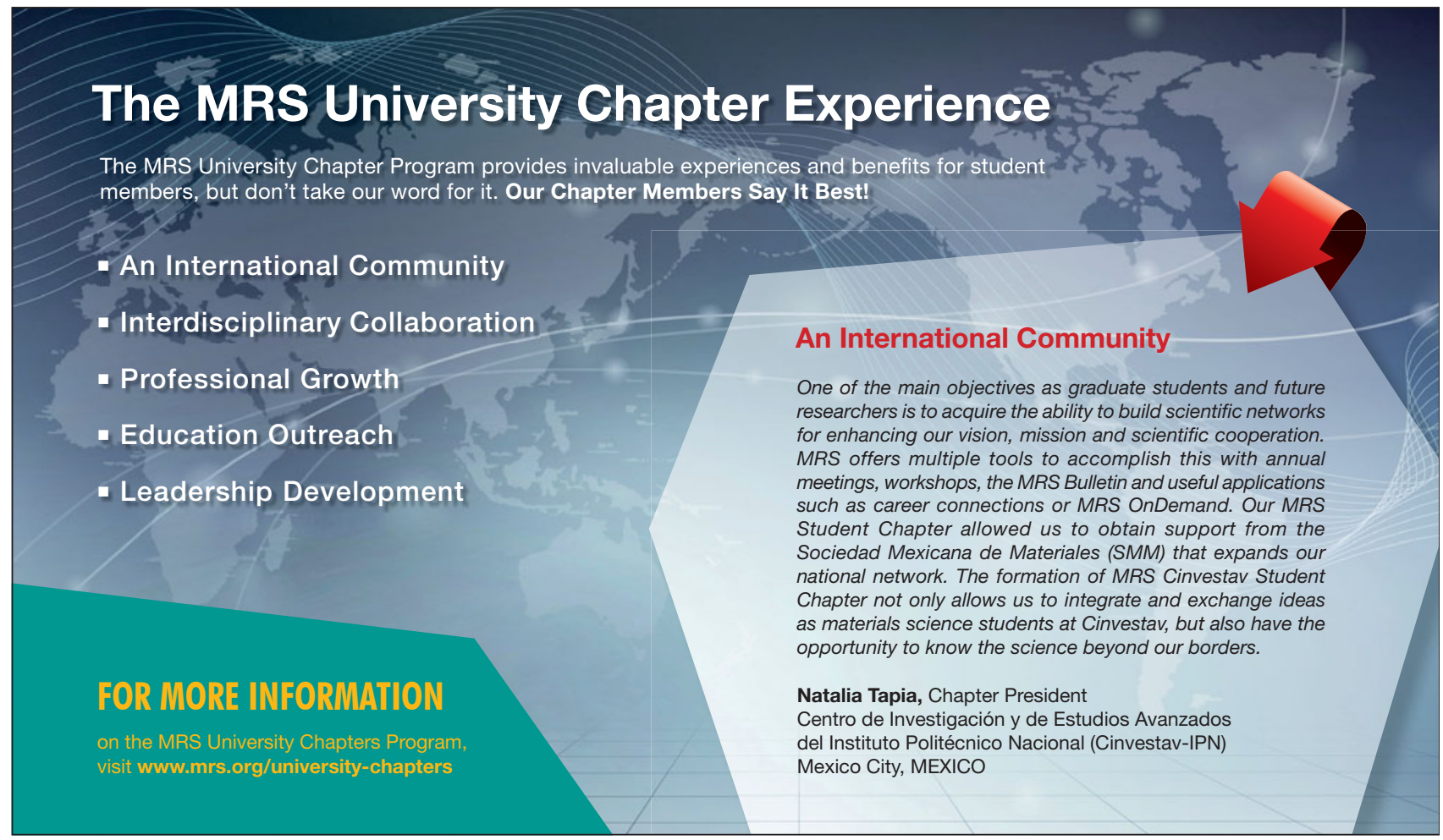

